\title{
Comparación de la normativa ambiental costarricense con países de América y Europa (España)
}

\author{
Wendy Villalobos-González' \& Rodolfo Antonio Hernández-ChaverRI ${ }^{2}$ \\ 1. Universidad Estatal a Distancia, Costa Rica. Encargada Cátedra de Ciencias Químicas. Unidad de Investigaciones \\ Bioenergéticas y Ambientales. Correo: wvillalobosg@uned.ac.cr \\ 2. Universidad Estatal a Distancia, Costa Rica. Encargado Cátedra de Ciencias Químicas para Ingeniería. Unidad de \\ Investigaciones Bioenergéticas y Ambientales. Correo: rohernandez@uned.ac.cr
}

Recibido: 03 de febrero de 2018

Aceptado: 04 de abril de 2018

\section{RESUMEN}

Costa Rica es identificada a nivel mundial como un país verde en términos de materia ambiental, por lo que sus gobernantes se han preocupado por tener reglamentos actualizados a los cambios internacionales y ser pioneros en la toma de decisiones ambientales. El presente documento muestra una comparación de las normativas ambientales de países suramericanos (Brasil y Ecuador), centroamericanos (Costa Rica, Panamá y Nicaragua), norteamericanos (México) y europeos (España). Se determinó que España es el país con valores límites máximos más exigentes que el resto de los países en estudio. Sin embargo, Costa Rica tiene normativa de agua, aire y suelo; con mayor cantidad de parámetros reglamentados. El país con mejor normativa es Nicaragua entre los estudiados. La falta de recursos y personal de las instituciones del gobierno costarricense para aplicar los reglamentos con mayor rigurosidad es una de las causas de la creciente contaminación ambiental en la zona urbana.

Palabras claves: normativa, ambiente, agua, aire, suelo.

\begin{abstract}
Comparison of Costa Rican environmental regulations with countries in America and Europe (Spain)

Costa Rica is identified worldwide as a green country in terms of environmental matters, so its leaders have been concerned to have updated regulations to international changes and be pioneers in making environmental decisions. This document shows a comparison of the environmental regulations of South American countries (Brazil and Ecuador), Central America (Costa Rica, Panama and Nicaragua), North Americans (Mexico) and Europeans (Spain). It was determined that Spain is the country with the highest limit values more demanding than the rest of the countries under study. However, Costa Rica has water, air and soil regulations; with a greater number of regulated parameters. The
\end{abstract}

country with the best regulations is Nicaragua among those studied. The lack of resources and personnel of the Costa Rican government institutions to apply the regulations with greater rigor is one of the causes of the growing environmental pollution in the urban area.

Key words: normative, environment, water, air, soil.

\section{Introducción}

Con la revolución industrial (1820-1840) y la tecnificación de la economía surgieron una serie de accidentes ambientales causados por la presencia de tóxicos o contaminantes descritos en el libro "Silent Spring" de Rachel Carson publicado en 1962. La obra inspiró la movilización ecologista en los Estados Unidos y consiguió que el Departamento de Agricultura prohibiera el dicloro difenil tricloroetano (DDT) y sentó las bases para la creación de la Agencia de Protección Ambiental de los Estados Unidos (EPA), (Burcham, 2014). A partir de este antecedente se genera un boom a nivel internacional por la creación de legislación ambiental y órganos reguladores.

Costa Rica ocupa un $0,03 \%$ de la superficie total terrestre $\left(51100 \mathrm{~km}^{2}\right)$ pero concentra cerca del $6 \%$ de la biodiversidad del mundo, es por esta razón que el estado costarricense ha creado leyes en pro del medio ambiente. Con la Ley Orgánica del Ambiente, decreto 7554 creada en 1995, el estado procura que los costarricenses obtengan los instrumentos necesarios para conseguir un ambiente sano y ecológicamente equilibrado. Es con este decreto que se crea el Ministerio de 
Ambiente y Energía (MINAE): órgano rector en ambiente, y la Secretaria Técnica Ambiental (SETENA): encargada de realizar evaluaciones de impacto ambiental a actividades humanas que alteren o destruyan elementos del ambiente o generen residuos tóxicos o peligrosos. (Ley Orgánica del Ambiente Decreto 7554, 1995)

Costa Rica cuenta con legislación en materia ambiental, que abarca desde la Constitución Política hasta las diferentes leyes, reglamentos y decretos; el problema se centra en la falta de recursos y personal de las instituciones del gobierno para aplicar los reglamentos con mayor rigurosidad y atender la demanda de todo el territorio nacional (Rojas \& García, 2008). En la tabla 1 se muestra un resumen de la principal legislación costarricense en materia ambiental.

Este documento tiene como objetivo realizar una comparación de la legislación ambiental costarricense, con otros países de América y
Europa, en términos de reglamentos para la gestión de los suelos, el agua y el aire. Cabe resaltar que el alcance de esta investigación se limita a la legislación existente en cada país, sin entrar en detalles sobre la implementación y ejecución de dicha legislación.

\section{Metodología}

\section{Escogencia de países para revisión} de legislación ambiental

Este estudio abarcó la legislación ambiental (incluyendo leyes, reglamentos y normas técnicas obligatorias) en tres países de Centroamérica: Nicaragua, Costa Rica y Panamá; dos países de Suramérica: Brasil y Ecuador; un país de Norteamérica: México; y un país europeo: España.

Para la selección de países en América del Sur, se tomó en cuenta la cantidad de muertes

TABLA 1

\section{Resumen de la legislación ambiental costarricense}

\begin{tabular}{ccl} 
Matriz & \multicolumn{1}{c}{ Título } & \multicolumn{1}{c}{ Objeto } \\
Agua & $N^{\circ}$ 276: Ley de Aguas (1996) & $\begin{array}{l}\text { Establece la legislación entorno a las aguas de uso } \\
\text { público y privado. }\end{array}$
\end{tabular}

Decreto $N^{\circ} 32327$ : Reglamento para la Calidad del Agua Potable. (2005)

Decreto N ${ }^{\circ} 33601$ : Reglamento de Vertido y Reuso de Aguas Residuales (2007)

Aire $\quad$ Decreto 36551: Reglamento sobre la Emisión de
Contaminantes Atmosféricos Provenientes de
Calderas y Hornos de tipo Indirecto (2011)
Calderas y Hornos de tipo Indirecto (2011)

Decreto 30221-S: Reglamento de Inmisión de Contaminantes Atmosféricos (2002)
Establece los niveles máximos que deben tener aquellos componentes del agua que pueden representar un riesgo para la salud humana.

Describe los lineamientos entorno al manejo de las aguas residuales vertidas o reusadas.

Posee la finalidad de controlar los niveles máximos de las emisiones al aire de las actividades industriales, comerciales y de servicios, en cuyos procesos requieran la operación de calderas y hornos de tipo indirecto.

Regular por medio de valores máximos permitidos las inmisiones al aire que puedan afectar la calidad del aire y por ende la salud de las personas.

Suelo Ley 29375: Uso, Manejo y Conservación del Suelo (1998)

Busca proteger, conservar y mejorar los suelos en gestión integrada y sostenible con los demás recursos naturales

Decreto 37757: Reglamento sobre Valores Guía en Suelos para descontaminación de Sitios Afectados por Emergencias Ambientales y Derrames (2005)

Establece el listado de sustancias químicas y los valores guía a partir de los cuales se podrían provocar alteraciones perjudiciales a la calidad del suelo y la salud

Decreto 18564, Creación del Servicio Nacional de Conservación del Suelo (1998)

Entidad encargada de ejecutar el Plan Nacional de Conservación del Suelo 
atribuidas a la contaminación del aire según un estudio realizado en los años 2004-2008 (Green, 2012); por lo que se seleccionó Brasil (país con más muertes atribuidas) y ecuador (menor cantidad de muertes). En Norteamérica, se incluyó a México ya que en este país se encuentra una de las ciudades más contaminadas del mundo: Ciudad de México, como lo reporta la Ambient Air Pollution Database. (OMS, 2016). En el continente europeo se consideró a España por ser ejemplo en materia ambiental a nivel mundial. España se encuentra en la posición número 6 de la lista del índice de Desempeño Ambiental 2016. Este índice evalúa datos como: salud, clima, energía, demanda y calidad del agua, sanidad, calidad del aire, bosques, agricultura, intensidad de pesca y áreas quemadas, ozono a nivel del suelo, contaminación de aire interno entre otros. Es importante indicar que Costa Rica se encuentra en la posición 54 en esta lista (Asociación Ambiente y Sociedad, 2017).

\section{Obtención de información y análisis}

La información se obtuvo a partir de una búsqueda bibliográfica de los reglamentos de cada país según la página de internet de los órganos rectores ambientales correspondientes, vigentes al mes de noviembre del año 2017. Con el fin de agrupar el análisis, se definieron tres criterios o temas que resultan claves en legislación ambiental y en la operación de cualquier actividad productiva, como lo es la gestión del agua, suelo y aire.
La información encontrada se analiza de manera cuantitativa, siguiendo la siguiente sistematización:

1. Definición de los temas ambientales a analizar por país, con base en el criterio técnico. Los temas definidos fueron: agua potable, aguas residuales, emisiones e inmisiones al aire y suelos.

2. Recopilación de la legislación ambiental de cada país, para lo cual se consultaron las bases de datos disponibles en internet de las principales instituciones de gobierno y se complementó con estudios de compilación en la materia. El detalle de la legislación compilada se muestra en la sección de "Referencias" del presente documento.

3. Sistematización de la información para confeccionar matrices por tema.

4. Evaluación de la legislación ambiental de cada país y análisis comparativos de los mismos.

\section{Discusión y resultados}

\section{Sobre órganos rectores \\ en Materia Ambiental}

Los países deben nombrar un órgano rector encargado de velar por el cumplimiento de la normativa ambiental, en la tabla 2 se observa el nombre del órgano rector de todos los países integrados en este estudio.

TABLA 2

Nombre de los órganos rectores en materia ambiental de cada país incluido en este documento

\begin{tabular}{llcl}
\multicolumn{1}{c}{ País } & \multicolumn{1}{c}{ Órgano rector ambiental } & Año de creación & \multicolumn{1}{c}{ Sitio web } \\
Costa Rica & Ministerio de Ambiente y Energía & 1995 & http://www.minae.go.cr/index.php/es/ \\
Brasil & Ministerio de Ambiente & 1992 & http://www.mma.gov.br/ \\
Ecuador & Ministerio de Ambiente & 1996 & http://www.ambiente.gob.ec/ \\
Panamá & Ministerio de Ambiente & 1998 & http://www.miambiente.gob.pa/ \\
Nicaragua & Ministerio del Ambiente y de los Recursos Naturales & 1994 & http://www.marena.gob.ni/ \\
México & Secretaria de Medio Ambiente y Recursos Naturales & 2000 & https://www.gob.mx/semarnat \\
España & Ministerio de Agricultura y Pesca, Alimentación y Medio Ambiente & 1996 & http://www.mapama.gob.es/es/ \\
\hline
\end{tabular}


En Costa Rica el MINAE es la institución encargada de administrar los recursos destinados a la protección del ambiente, su misión es "contribuir al mejoramiento de la calidad de vida de las y los habitantes mediante la promoción del manejo, conservación y desarrollo sostenible de los elementos, bienes, servicios y recursos ambientales y naturales del país" (Ministerio de Ambiente y Energía, 2017).

\section{Reglamentos para la gestión del aire}

Costa Rica tiene dos reglamentos principales que regulan la calidad del aire: el Reglamento sobre la Emisión de Contaminantes Atmosféricos Provenientes de Calderas y Hornos de tipo Indirecto (Decreto 36551) y el Reglamento sobre Inmisión de Contaminantes Atmosféricos (Decreto 30221-S).

El Reglamento sobre la Emisión de Contaminantes Atmosféricos Provenientes de Calderas y Hornos de tipo Indirecto, posee la finalidad de controlar los niveles máximos de las emisiones al aire de las actividades industriales, comerciales y de servicios, en cuyos procesos requieran la operación de calderas y hornos de tipo indirecto. Un horno o caldera, es una estructura dedicada a la producción de vapor de agua, con energía calórica a partir de combustibles fósiles o biomásicos; dentro de los combustibles fósiles se pueden nombrar el bunker, diésel, gasóleo, keroseno o gas natural (GLP). En el reglamento se clasifica las calderas de acuerdo con la cantidad de vapor de agua producida y sus dimensiones y a los hornos de acuerdo con su capacidad de producción de vapor, el área de calefacción y su potencia. (Reglamento sobre la Emisión de Contaminantes Atmosféricos Provenientes de Calderas y Hornos de tipo Indirecto, 2011).

Los parámetros regulados por los reglamentos son principalmente tres: partículas totales en suspensión (PTS), dióxido de azufre $\left(\mathrm{SO}_{2}\right)$ y óxidos de nitrógeno $\left(\mathrm{NO}_{\mathrm{X}}\right)$. Los valores máximos permitidos dependen del combustible empleado y de la potencia de la caldera u horno. En la tabla 3 se muestran los valores máximos permitidos según el reglamento de cada país.
La regulación ecuatoriana y la mexicana, además de los parámetros ya mencionados regulan el monóxido de carbono como se muestra en la tabla 4.

En Panamá el decreto No5 "Normas Ambientales de Emisiones de Fuentes Fijas", indica como fuente fija de combustión significativa la que tiene una potencia calorífica igual o mayor a tres millones de vatios $\left(3 \times 10^{6} \mathrm{~W}\right)$ o diez millones de unidades térmicas británicas por hora $\left(10 \times 10^{6} \mathrm{BTU} / \mathrm{h}\right)$ y los parámetros los define de acuerdo con la actividad y código CIIU, como se muestra en la tabla 5. Según sea la carga de contaminante en toneladas/día, así será la frecuencia de caracterización del efluente gaseoso. (Normas Ambientales de Emisiones de Fuentes Fijas de Panamá, 2009)

En el caso de Nicaragua no se encontró una norma para emisión de contaminantes atmosféricos de fuentes fijas; solo de fuentes móviles y calidad del aire (inmisión de contaminantes).

Para el reglamento costarricense es una deficiencia el no tomar en cuenta la emisión de monóxido de carbono, este contaminante es transformado en la atmosfera en dióxido de carbono; el cual es un gas clasificado como de efecto invernadero. Como parte de una combustión incompleta en calderas y hornos se puede aumentar su concentración causando serios efectos ambientales, un adecuado control de la temperatura y la cantidad de oxígeno, puede disminuir la concentración de monóxido de carbono formado. (Abarca, 2007).

Los criterios utilizados para limitar las emisiones al aire en cada país son muy heterogéneos en los lineamientos a seguir, por ejemplo, mientras en países como Costa Rica, México, España y Brasil se definen los criterios en base a la capacidad de la fuente fija (caldera u horno), en Ecuador se utiliza un único argumento (potencia calorífica de la fuente sea mayor a $3 \times 10^{6} \mathrm{~W}$ ) y en Panamá se define los valores máximos permisibles de acuerdo con la actividad productiva, lo cual es un acierto de esta metodología debido las características del efluente varía de acuerdo con el tipo de industria, proceso productivo y capacidad productiva. 
TABLA 3

Valores de emisión de contaminantes atmosféricos permitidos por la legislación costarricense, brasileña, ecuatoriana, mexicana y española

\begin{tabular}{|c|c|c|c|c|}
\hline País & Tipo de combustible & PTS $\left(\mathrm{mg} / \mathrm{m}^{3}\right)$ & $\mathrm{NO}_{\mathrm{x}}\left(\mathrm{mg} / \mathrm{m}^{3}\right)$ & $\mathrm{SO}_{2}\left(\mathrm{mg} / \mathrm{m}^{3}\right)$ \\
\hline \multirow[t]{6}{*}{ Costa Rica } & Bunker & \multirow{5}{*}{$135-175$} & $550-600$ & \multirow{6}{*}{2500} \\
\hline & Diésel & & $200-220$ & \\
\hline & Gasóleo & & 420 & \\
\hline & Keroseno & & 150 & \\
\hline & GLP & & 170, C,D: exento & \\
\hline & Biomasa & A: 120, B: 150, C,D: 175 & 650 & \\
\hline \multirow[t]{5}{*}{ Brasil } & Gasóleo & $300-100$ & - & No presenta \\
\hline & Madera & $130-730$ & 650 & No presenta \\
\hline & Gas natural & No presenta & $200-320$ & No presenta \\
\hline & Biomasa & $200-380$ & 350 & No presenta \\
\hline & Gas de refinería & $50-150$ & $200-320$ & No presenta \\
\hline \multirow[t]{4}{*}{ Ecuador } & Sólido & 150 & 850 & 1650 \\
\hline & Bunker & 150 & 550 & 1650 \\
\hline & Diésel & 100 & 500 & 1000 \\
\hline & GLP & No presenta & 140 & No presenta \\
\hline \multirow[t]{4}{*}{ España } & Biomasa & $30-50$ & $200-400$ & 200 \\
\hline & Líquidos & $30-50$ & $200-400$ & $200-850$ \\
\hline & Gaseoso en general & 5 & 200 & 35 \\
\hline & Gas licuado & No presenta & $100-150$ & 5 \\
\hline \multirow[t]{3}{*}{ México } & Sólido & 60 & 110 & 550 \\
\hline & Líquido & $60-75$ & $110-190$ & 550 \\
\hline & Gas & No presenta & $110-190$ & No presenta \\
\hline
\end{tabular}

TABLA 4

Valores máximos permitidos de monóxido de carbono en la norma ecuatoriana y mexicana para emisiones de fuentes fijas

\begin{tabular}{cc|cc|}
\multicolumn{2}{|c|}{ Ecuador } & \multicolumn{2}{c}{ México } \\
Tipo de combustible & CO $\left(\mathrm{mg} / \mathrm{m}^{3}\right)$ & Capacidad térmica del equipo $\mathrm{GJ} / \mathrm{h}$ & $\mathrm{CO}\left(\mathrm{mg} / \mathrm{m}^{3}\right)$ \\
\hline Solido & 1800 & Mayor de 0.53 a 5.3 & L: $400 \mathrm{G}: 400$ \\
Bunker & 300 & Mayor de 5.3 a 42.4 & L: 400 G: 400 \\
Diésel & 200 & Mayor de 42.4 a 106 & L: 400 G: 400 \\
GLP & 100 & Mayor de 106 a 530 & Sy L: 400 G: 400 \\
& & Mayor de 530 & Sy L: 400 G: 400 \\
\hline
\end{tabular}

En el caso de la reglamentación en términos de calidad del aire, Costa Rica cuenta con el Reglamento de Inmisión de Contaminantes
Atmosféricos decreto 30221-S, el mismo tiene como objetivo principal el de regular por medio de valores máximos permitidos las inmisiones al 
TABLA 5

Límites máximos permitidos por actividad CIIU, según norma Ambiental Panameña

\begin{tabular}{|c|c|c|c|}
\hline Actividad CIIU & $\begin{array}{c}\text { PTS } \\
\left(\mathrm{mg} / \mathrm{m}^{3}\right)\end{array}$ & $\mathrm{SO}_{2}\left(\mathrm{mg} / \mathrm{m}^{3}\right)$ & $\mathrm{NO}_{x}\left(\mathrm{mg} / \mathrm{m}^{3}\right)$ \\
\hline Producción de Cemento (3692) & 50 & 400 & 600 \\
\hline Fundiciones (3720) & 20 & - & - \\
\hline Molinos de Papel (3419) & 100 & - & $2 \mathrm{Kg} / \mathrm{t}$ \\
\hline Ingenios Azucareros (3131) & 100 & 2000 & $\begin{array}{l}\text { Combustibles líquidos: } 450 \\
\text { Combustibles sólidos: } 750\end{array}$ \\
\hline Generación Termoeléctrica (4101) & 50 & $\begin{array}{c}0.2 \mathrm{tpd} / \mathrm{MW} \text { (hasta } 500 \mathrm{MW} \text { ) } \\
0.1 \mathrm{tpd} / \mathrm{MW} \text { (incrementos arriba de } 500 \mathrm{MW} \text { ) }\end{array}$ & $\begin{array}{c}\text { Carbón: } 750 \text { Petróleo: } 460 \\
\text { Gas: } 320\end{array}$ \\
\hline Generación Termoeléctrica con Turbinas de Gas (4101) & & No se puede exceder $2000 \mathrm{mg} / \mathrm{Nm}^{3}$ ni $500 \mathrm{tpd}$ & \\
\hline Fabricación de Productos Petroquímicos & 20 & 500 & 300 \\
\hline Refinación de Petróleo & 50 & $\begin{array}{l}150 \text { (unidades con recuperación de azufre) } \\
500 \text { (unidades de combustión) }\end{array}$ & 460 \\
\hline Fabricación de Productos Farmacéuticos & 20 & - & - \\
\hline Procesamiento de Aceite Vegetal & 50 & - & - \\
\hline Otras Actividades & 100 & 2000 & $\begin{array}{c}\text { Carbón: } 750 \text { Petróleo: } 460 \\
\text { Gas: } 320\end{array}$ \\
\hline
\end{tabular}

aire que puedan afectar la calidad del aire y por ende la salud de las personas. Según el decreto deben realizar análisis de los parámetros (ver tabla 6) las empresas que por su sistema productivo genera alguno de los contaminantes o por indicación del Ministerio de Salud (Costa Rica) en caso de denuncia ambiental. En la tabla 6, se observa los parámetros a medir según las distintas regulaciones de los países incluidos en este documento.

Es importante destacar que a la fecha Panamá no cuenta con un reglamento de calidad del aire (inmisiones) dentro de las normas de calidad ambiental del Ministerio de Ambiente. La autoridad del Canal de Panamá (ACP) tiene su propia norma basada en limites reportados por la EPA, la División de Administración Ambiental basada en la Directriz: AD-2003-02, estableció el Programa de Control de Emisiones a la Atmósfera, para proyectos, obras o actividades que realice la ACP o terceros en áreas de propiedad de la ACP y en áreas bajo su administración privativa. (Autoridad del Canal de Panamá (ACP), 2006)

Al analizar los datos de la tabla 6, España tiene valores permisibles bajos en comparación con los otros países, la Norma Real Decreto 102/2011 toma en cuenta inmisiones como media aritmética anual de otras sustancias como benceno $\left(5 \mu \mathrm{g} / \mathrm{m}^{3}\right)$, arsénico $\left(6 \mathrm{ng} / \mathrm{m}^{3}\right)$, cadmio (5 ng/ $\left.\mathrm{m}^{3}\right)$, níquel $\left(20 \mathrm{ng} / \mathrm{m}^{3}\right)$ y benzo pireno $\left(1 \mathrm{ng} / \mathrm{m}^{3}\right)$; sustancias que no se contemplan en otros países. Quizás estas sean las razones del porque España se encuentra en la posición número 6 de la lista del índice de Desempeño Ambiental 2016 solo por debajo de países como Finlandia, Islandia, Suecia, Dinamarca y Eslovenia (Asociación Ambiente y Sociedad, 2017).

Es importante destacar que en Costa Rica una fortaleza del reglamento de inmisión es el tomar en cuenta parámetros como cloruro de hidrógeno, fluoruro de hidrógeno, formaldehído, plomo, amoniaco, sulfuros de hidrógeno e hidrocarburos totales, mismos que no son reportados en los otros países de esta comparación. Sin embargo, una debilidad de nuestro reglamento es el no considerar las partículas menores a 2,5 $\mu \mathrm{m}\left(\mathrm{PM}_{2,5}\right)$, valor si reportado en los otros reglamentos.

Los países al menos de una misma región deberían adoptar un conjunto armonizado de estándares de calidad del aire para proteger la 
TABLA 6

Valores permisibles de inmisión de contaminantes atmosféricos según cada país

\begin{tabular}{|c|c|c|c|c|c|c|c|}
\hline \multirow[b]{2}{*}{ Parámetro } & \multirow[b]{2}{*}{ Periodo } & \multicolumn{6}{|c|}{ País } \\
\hline & & $\begin{array}{l}\text { Costa Rica } \\
\text { Decreto } \\
\text { No. 30221-S }\end{array}$ & $\begin{array}{l}\text { Nicaragua } \\
\text { Norma } \\
\text { № } 211\end{array}$ & $\begin{array}{c}\text { Brasil } \\
\text { RESOLUÇÃO } \\
\text { CONAMA n } 3\end{array}$ & $\begin{array}{c}\text { Ecuador } \\
\text { Acuerdo } N^{\circ} 50\end{array}$ & $\begin{array}{c}\text { México } \\
\text { NOM-035- } \\
\text { SEMARNAT-1993 }\end{array}$ & $\begin{array}{c}\text { España } \\
\text { Real Decreto } \\
\text { 102/2011 }\end{array}$ \\
\hline \multirow{2}{*}{$\begin{array}{l}\text { Partículas totales en } \\
\text { suspensión }\left(\mu \mathrm{g} / \mathrm{m}^{3} \mathrm{PTS}\right)\end{array}$} & Anual & 90 & 75 & 80 & $1 \mathrm{mg} / \mathrm{cm}^{2}$ & 75 & No presenta \\
\hline & $24 \mathrm{~h}$ & 240 & 260 & 240 & No presenta & 260 & No presenta \\
\hline \multirow{2}{*}{$\begin{array}{l}\text { Partículas }>10 \mu \mathrm{m} \\
\left(\mu \mathrm{g} / \mathrm{m}^{3} \mathrm{PM}_{10}\right)\end{array}$} & Anual & 50 & 50 & 50 & 50 & 40 & 40 \\
\hline & $24 \mathrm{~h}$ & 150 & 150 & 150 & 100 & 75 & 50 \\
\hline \multirow{2}{*}{$\begin{array}{l}\text { Dióxido de azufre } \\
\left(\mu \mathrm{g} / \mathrm{m}^{3} \mathrm{SO}_{2}\right)\end{array}$} & Anual & 80 & 80 & 80 & 60 & 67 & 20 \\
\hline & $24 \mathrm{~h}$ & 365 & 365 & 365 & 125 & 293 & 125 \\
\hline \multirow{2}{*}{$\begin{array}{l}\text { Monóxido de carbono } \\
\left(\mathrm{mg} / \mathrm{m}^{3} \mathrm{CO}\right)\end{array}$} & $8 \mathrm{~h}$ & 10 & 10 & 10 & 10 & No presenta & 10 \\
\hline & $1 \mathrm{~h}$ & 40 & 40 & 40 & 30 & $13 \mu \mathrm{g} / \mathrm{m}^{3}$ & No presenta \\
\hline \multirow{2}{*}{$\begin{array}{l}\text { Dióxido de nitrógeno } \\
\left(\mu \mathrm{g} / \mathrm{m}^{3} \mathrm{NO}_{\mathrm{x}}\right)\end{array}$} & Anual & 100 & 100 & 100 & 40 & No presenta & 40 \\
\hline & $1 \mathrm{~h}$ & 400 & 400 & 320 & 200 & 384 & 200 \\
\hline Ozono $\left(\mu \mathrm{g} / \mathrm{m}^{3} \mathrm{O}_{3}\right)$ & $1 \mathrm{~h}$ & 160 & 235 & 160 & $100(8 h)$ & $140(8 \mathrm{~h})$ & 120 \\
\hline Plomo $\left(\mu \mathrm{g} / \mathrm{m}^{3} \mathrm{~Pb}\right)$ & Anual & 0,5 & 1,5 & No presenta & No presenta & 1,5 & 0,5 \\
\hline \multirow{2}{*}{$\begin{array}{l}\text { Hidrocarbur totales } \\
\left(\mu \mathrm{g} / \mathrm{m}^{3} \mathrm{CH}_{4}\right)\end{array}$} & Anual & No presenta & No presenta & 60 & No presenta & No presenta & No presenta \\
\hline & $3 \mathrm{~h}$ & 160 & No presenta & 150 & No presenta & No presenta & No presenta \\
\hline
\end{tabular}

salud pública, con objetivos intermedios que dependan de las circunstancias particulares nacionales y/o locales (como se sugiere en las Guías de Calidad del Aire de la OMS). Esto facilitaría la elaboración de reportes, análisis, y evaluaciones comparativas a nivel regional, así como el inicio del fortalecimiento de un enfoque regional en el mejoramiento de la calidad del aire (Green, 2012).

\section{Reglamentos para la gestión del agua}

Los componentes o características del agua pueden representar un riesgo para la salud e inconvenientes para su preservación, es por esta razón que se crea el Reglamento para la Calidad del Agua Potable.

La tabla 7, muestra los valores máximos permitidos para los parámetros a regular en los distintos reglamentos según cada país en estudio. Al realizar la comparación de los parámetros para la calidad del agua potable, se observa, cómo tanto los parámetros como los valores de estos son parecidos y por lo tanto casi no varían de un país a otro. La calidad del agua no es solo es una política de cada país sino una política internacional, donde la preocupación por el acceso de agua potable de buena calidad se ve reflejada en la normativa.

La Organización Mundial de la Salud (OMS) recomienda una concentración máxima de nitrato de $50 \mathrm{mg} / \mathrm{L}$, ya que una alta concentración de nitrato en el agua potable puede causar alta concentración de metahemoglobina (hemoglobina con hierro asociado) en sangre; lo que genera una reducción en la capacidad de los glóbulos rojos para liberar oxígeno en los tejidos. Los grupos etarios especialmente susceptibles a la formación de metahemoglobina son jóvenes infantes, niños y mujeres embarazadas (OMS, 2003) Es por esta razón que países como Brasil, México y Panamá tienen valores máximos permitidos para este ión de $10 \mathrm{mg} / \mathrm{L}$, en comparación con países como Costa Rica, Nicaragua, Ecuador y España, donde el limite supera los $25 \mathrm{mg} / \mathrm{L}$.

El cloro libre en el agua potable no es particularmente tóxico para los humanos, sin embargo, es la principal fuente de la exposición al cloro por lo que el valor máximo debe ser de 


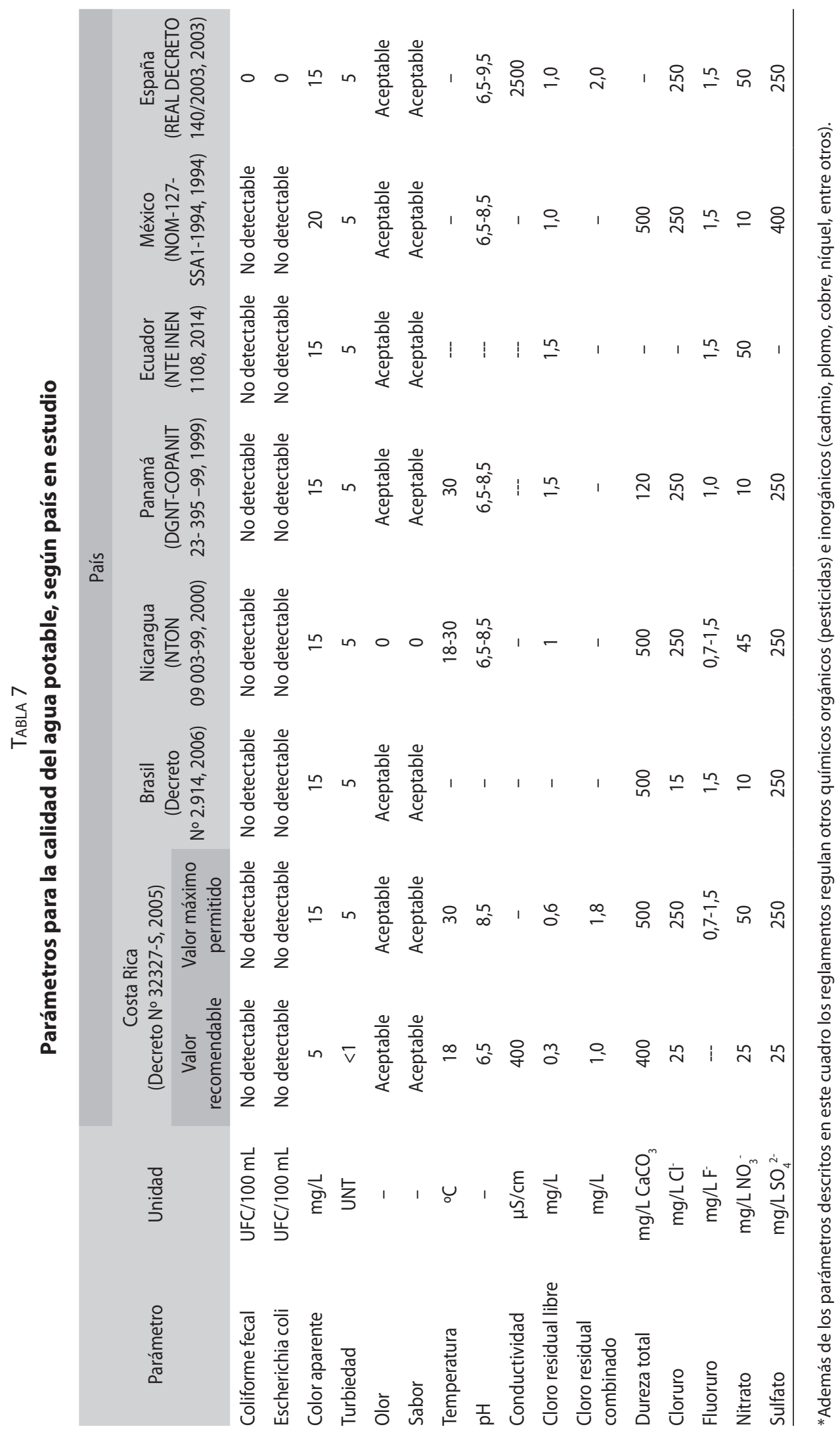


$5 \mathrm{mg} / \mathrm{L}$ por la suma de ácido hipocloroso e hipoclorito. Los valores máximos permitidos para este parámetro en todas las normas de calidad de agua potable consultadas se encuentran por debajo de este valor, esto porque si hay presencia de sustancias húmicas se pueden formar trihalometanos en presencia de cloro como: cloroformo, bromoformo, bromodiclorometano o dibromoclorometano; sustancias que tienen un efecto toxico biológico (OMS, 2003).

En términos de agua no solo la calidad del agua potable es una preocupación; sino también el saneamiento de las aguas residuales. El decreto costarricense $\mathbf{N}^{\circ}$ 33601: Reglamento de vertido y reusó de aguas residuales, describe los lineamientos entorno al manejo de las aguas residuales vertidas o reusadas. La tabla 8 , indica los valores máximos permitidos para los diferentes parámetros medidos para el vertido de aguas residuales a un cuerpo receptor con el fin de disminuir la contaminación de estos. La contaminación de los cuerpos de agua favorece la proliferación de enfermedades de transmisión hídrica, reduce el número de fuentes disponibles, eleva los costos para el abastecimiento de agua para consumo humano y pone en peligro de extinción a muchas especies de flora y fauna (MINAE, 2007).

En la tabla 8, no se observan valores máximos de emisión para Brasil porque no cuenta con un reglamento. Según un artículo de un diario local, los brasileños producen 32 millones de metros cúbicos de desechos de alcantarillado por día, de los cuales sólo 14 millones se trasladan y solamente 4,8 millones son tratados en plantas de tratamiento. La diferencia de metros cúbicos de desechos es descartada en los ríos. El periodista indica que los números ilustran el tamaño del problema "Los ríos de Brasil, en especial en los grandes centros urbanos, se están muriendo; el gran problema del área de saneamiento es la falta de regulación" (Verdélio, 2017, p.40). Brasil no cuenta con una reglamentación sobre el vertido y reusó de aguas residuales industriales o domésticas "Es un juego que no hay reglas, ni juez, no hay ley, ni un órgano regulador" (Verdélio, 2017, p.40).

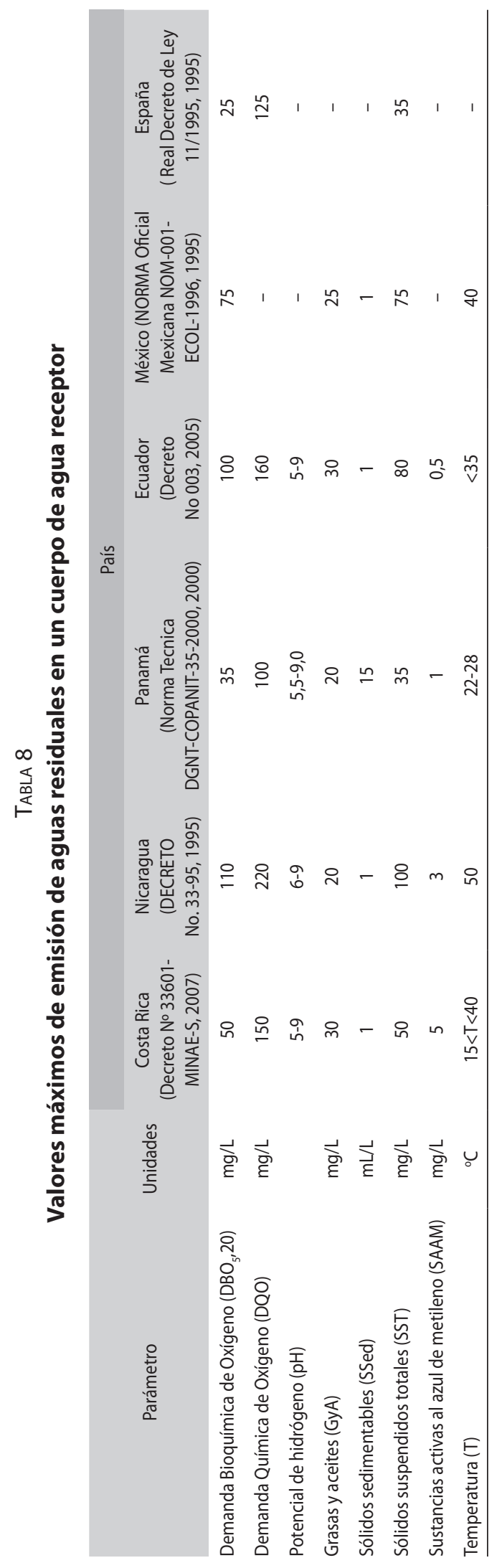


Adicional a los parámetros descritos en la tabla 8 , el reglamento costarricense solicita realizar análisis extras dependiendo del tipo de agua proveniente por actividad según código CIIU. Por ejemplo, actividades que involucren ganadería, agricultura y crianza de animales, deben realizar análisis de nitrógeno total y fosfatos en sus aguas residuales.

España tiene valores de emisión de aguas residuales a cuerpos receptores más bajas, esto debido a que en Europa la depuración de aguas residuales urbanas es una práctica obligada desde la publicación de la Directiva 91/271/CE. El cumplimiento de este objetivo ha propiciado la construcción y puesta en marcha de instalaciones, que garanticen la recogida y tratamiento de aguas. Sin embargo, sigue pendiente un número importante de instalaciones por construir, para garantizar los requerimientos mínimos exigidos por la Directiva (Instituto Nacional del Medio Ambiente, 2017).

Nicaragua tiene valores de emisión más altos; además de ello solo el 12,5\% de las aguas residuales son tratadas y en las zonas rurales no existe tratamiento. Estas son las principales causas de contaminación de los ríos en este país (Calero, M., 2017).

En Costa Rica la "Política Nacional de Saneamiento en Aguas Residuales" (PNSAR) se constituye en la propuesta de intervención del Estado y sus instituciones para resolver el problema de las aguas residuales. A partir del censo llevado a cabo en el año 2016 por el Instituto Nacional de Estadística y Censos (INEC) del total de 1436120 viviendas del país, 307718 $(21,43 \%)$ tienen conexión a alcantarillado o cloaca, y 1097531 (76,42\%) están conectadas a tanque séptico. La población de Costa Rica en el 2015, según datos de INEC, era de 4832 227 habitantes, y Acueductos y Alcantarillados (AyA) estima que la generación diaria de aguas residuales por persona es de $0,2 \mathrm{~m}^{3} /$ día, de este total de las aguas residuales solo el $8 \%$ son tratadas (Llorca, 2017).

A pesar de este Reglamento de Vertido y Reúso de Aguas Residuales, muchos de los entes generadores no presentan reportes operacionales ante el Ministerio de Salud, que brinden información del estado y funcionamiento de los sistemas de tratamiento o no lo están haciendo con la periodicidad establecida en la legislación vigente solamente para cumplir. La falta de recursos y personal de este y otros ministerios es una realidad (Rojas \& García, 2008) por lo que las nuevas políticas en el ámbito ambiental deberían de fortalecer las entidades que tienen a cargo el control y la vigilancia de las actividades productivas en pro del ambiente.

\section{Reglamentos para la gestión del suelo}

En torno a la gestión del componente suelo, Costa Rica cuenta con el Servicio Nacional de Conservación del Suelo entidad creada por el decreto 18564; encargada de ejecutar el Plan Nacional de Conservación del Suelo y la Ley 7779: uso, manejo y conservación del suelo; que busca proteger, conservar y mejorar los suelos en gestión integrada y sostenible con los demás recursos naturales y el Decreto 37757: Reglamento sobre valores guía en suelos para descontaminación de sitios afectados por emergencias ambientales y derrames, donde se establece el listado de sustancias químicas y los valores guía a partir de los cuales se podrían provocar alteraciones perjudiciales a la calidad del suelo y la salud. La tabla 9, muestra la comparación de los valores guía de los distintos países, excluyendo a Nicaragua y Brasil ya que no se encontró reglamentación vigente.

En la tabla 9 se reporta el valor de Intervención el cual se define como la concentración de determinada sustancia en el suelo por encima del cual existen riesgos potenciales, directos o indirectos, a la salud humana, considerando un escenario de exposición genérica e indica la necesidad de acciones correctivas para proteger a los receptores. (Decreto $\mathrm{N}^{\circ}$ 37757-S, 2013). El reglamento sobre Valores Guía en Suelos para Descontaminación de Sitios Afectados por Emergencias Ambientales y Derrames (Decreto Ejecutivo 37757-S) estableció una serie de reglas especiales de responsabilidad por daño ambiental, que antes del año 2013 no había regulación al respecto. 


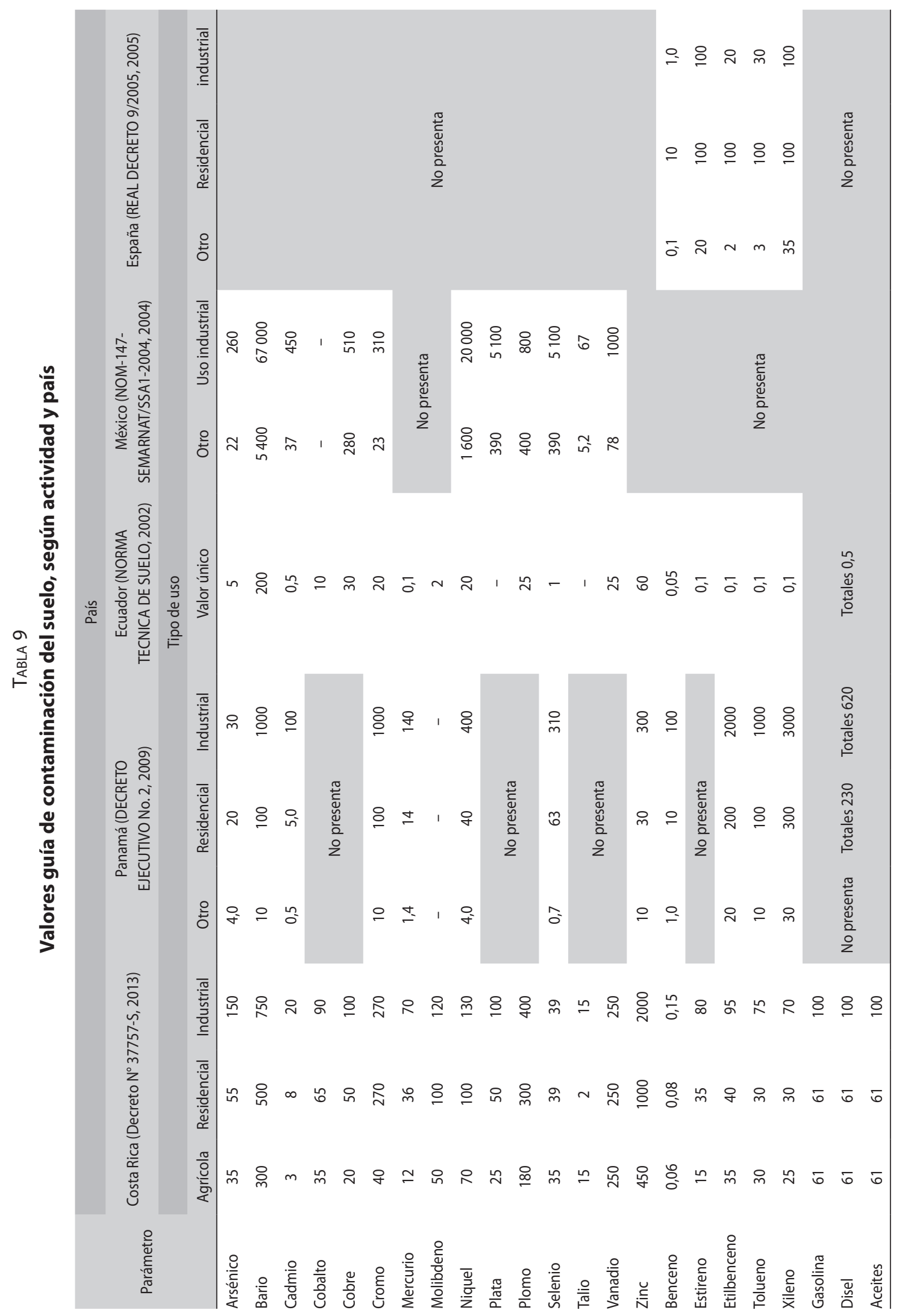


Entre los valores máximos permitidos para suelos contaminados, Costa Rica tiene un reglamento amplio en cuanto a la cantidad de parámetros regulados en comparación con los otros países. A pesar de esto los valores guía son considerablemente más altos, por ejemplo, el mercurio es 8,6 veces mayor que en Panamá.

\section{Conclusiones}

Según la comparación realizada de las normativas en materia ambiental de los países incluidos en este documento, España es el país con valores máximos permitidos más bajos en emisiones e inmisiones al aire y en vertido de aguas residuales. Costa Rica es el país con mayor cantidad de parámetros incluidos en las distintas normativas ambientales; prueba de ello es la cantidad de parámetros medidos en inmisión de contaminantes atmosféricos y en los valores guía para la contaminación del suelo. Nicaragua necesita de mayor cantidad de normativa ambiental, ya que no cuenta con normativa de emisión de contaminantes atmosféricos y sobre contaminación de suelos, que regule a los entes generadores.

Los esfuerzos realizados hasta la fecha en materia ambiental según lo expuesto a lo largo de este documento han propiciado que Costa Rica sea apreciado internacionalmente como un país verde o en pro de los recursos naturales, sin embargo, hace falta mayores esfuerzos para reforzar las entidades estatales que velan por el cumplimiento de la legislación.

La falta de recursos económicos y de personal de las instancias encargadas de la aplicación de la normativa nacional ambiental, implica que se acreciente la contaminación antropogénica. Por lo que, el fortalecimiento de las instituciones debe ser una prioridad de las nuevas políticas en materia ambiental, con el fin de fortalecer la fiscalización de las actividades productivas y su liberación y posible afectación al agua, aire y suelo.

\section{Recomendaciones}

La legislación ambiental regulatoria para evitar la contaminación debería de conformarse por actividad productiva, debido a que las emisiones al aire, los efluentes al agua y los residuos generados que podrían presentar un impacto ambiental potencial, depende del tipo de industria o comercio. Además de ello es necesario la articulación por ubicación geográfica para unificar criterios y valores máximos tolerables.

\section{Referencias}

Abarca, P. D. (2007). Potencia y Rendimiento de una Caldera. Chile. Asociación Chilena de Seguridad.

Asociación Ambiente y Sociedad. (2016). Indice de desempeño ambiental 2016. Recuperado de: http://www.ambienteysociedad.org.co/es/ cual-es-el-indice-de-desempeno-ambiental-2016/

Autoridad del Canal de Panamá (ACP). (2006). 2610ESM-109. Norma de calidad del aire ambiente. Recuperado de: https://micanaldepanama.com/wpcontent/uploads/2012/06/compras/109.pdf

Burcham, P. (2014). An Introduction to Toxicology. London. Springer-Verlang.

Calero, M. (24,03,2017). Agua residual se va a los ríos en Nicaragua. Diario La Prensa.

Dirección General de Salud Ambiental. (1994). NOM127-SSA1-1994. Agua para uso y consumo humano - Limites permisibles de calidad y tratamientos a que debe someterse el agua para su potabilizacion. Ciudad: México.

Jefatura del Estado Español. Real Decreto de Ley 11/1995. (1995). Normas apficables al tratamiento de las aguas residuales urbanas. Ciudad: España

Decreto Ejecutivo No. 2. (2009). Norma Ambiental de Calidad de Suelos para diversos usos. Panamá.

Decreto $\mathrm{N}^{\circ}$ 37757-S. (2013). Reglamento sobre valores guía en suelos para descontaminación de sitios afectados por emergencias ambientales y derrames. Costa Rica. 
Decreto No 003. (2005). Norma técnica para el control de descargas líquidas. Ecuador.

Decreto No 2.914. (2006). Dispõe sobre os procedimentos de controle e de vigilância da qualidade da água para consumo humano e seu padrão de potabilidade. Brasil.

Decreto No 32327-S. (2005). Reglamento para la Calidad del Agua Potable. Costa Rica.

Decreto $\mathrm{N}^{\circ}$ 33601-MINAE-S. (2007). Reglamento de Vertido y Reuso de Aguas Residuales. Costa Rica.

Decreto No. 33-95. (1995). Disposiciones para el control contaminacion provenientes descargas de aguas residuales domesticas, industriales y agropecuarias. Nicaragua.

DGNT-COPANIT 23- 395 -99. (1999). Agua potable. Panamá.

Green, J. S. (2012). La Calidad del aire en America Latina: Una visión panorámica. Washington D.C. Clean Air Institute.

Instituto Ecuatoriano de Normalización. (2014) Norma Tecnica Ecuatoriana NTE INEN 1108: Agua potable. Requisitos. Ecuador.

Instituto Ecuatoriano de Normalización. (2000) Norma Tecnica Ecuatoriana NTON 09 003-99. Normas tecnicas para el diseño de abastecimiento y potabilización del agua .

Instituto Nacional del Medio Ambiente. (2017). El Tratamiento de Aguas Residuales y su Marco Normativo. Recuperado de http://www.ismedioambiente.com/agenda/el-tratamiento-de-aguas-residuales-y-su-marco-normativo

Ley Organica del ambiente Decreto 7554. (1995). Recuperado de http://www.oas.org/dsd/fida/laws/legislation/costa_rica/costa_rica_7554.pdf

Llorca, F. G. (2017). Política Nacional de Saneamiento en Aguas Residuales 2016-2045. AyA, MINAE y MS.

Ministerio de Ambiente y Energía de Costa Rica (MINAE). Recuperado de http:/www.minae.go.cr/index.php/ es/2012-06-08-20-19-22/mision-y-vision

Norma Oficial Mexicana NOM-147-SEMARNAT/SSA12004. (2004). Criterios para determinar los valores de remediación en suelo. México.
Norma Oficial Mexicana NOM-001-ECOL-1996. (1995). Límites máximos permisibles de contaminantes en las descargas de aguas residuales en aguas y bienes nacionales. México.

Norma Oficial Mexicana NOM-085-SEMARNAT. (2011). Recuperado de http://www.dof.gob.mx/normasOficiales/4632/semarnat/semarnat.htm

Norma tecnica de suelo. (2002). Recuperado de file:///C:/ Users/Jorge/Downloads/pnt_suelo_20130923.pdf

Norma Tecnica DGNT-COPANIT-35-2000. (2000). Descarga de efluentes líquidos directamente a cuerpos de agua superficiales y subterraneas. Panamá.

Norma Tecnica Ecuatoriana para Emisiones a la Atmósfera de fuentes fijas. (2003). Recuperado de file:///C:/ Users/Jorge/Downloads/pnt_control_emision_atmos_ fuente_fija\%20(1).pdf

Normas Ambientales de Emisiones de Fuentes Fijas de Panamá. (2009). Recuperado de http://journals.plos. org/plosone/article/file?type=supplementary \&id=info :doi/10.1371/journal.pone.0088784.s008

Organización Mundial de la Salud (OMS). (2003). Guías para la calidad del agua potable de la OMS. Constituyentes inorgánicos y parámetros estéticos. Suiza.

Organización Mundial de la Salud (OMS). (2016). WHO's Urban Ambient Air Pollution database. Recuperado de http://www.who.int/airpollution/data/cities/en/

REAL DECRETO 140/2003. (2003). Criterios sanitarios de la calidad del agua de consumo humano. España.

REAL DECRETO 9/2005. (2005). Real Decreto 9/2005 de Actividades Potencialmente Contaminantes del Suelo. Recuperado de http://www.cepco.es/noticia. asp?id_rep $=1370$

Reglamento sobre la Emisión de Contaminantes Atmosféricos Provenientes de Calderas y Hornos de tipo Indirecto. (2011). San José, Costa Rica. Obtenido de http://www.digeca.go.cr/sites/default/files/reglamento_emision_contaminantes_atmosfericos_de_calderas_hornos_tipo_indirecto_0.pdf

Reglamento sobre la Inmisión de Contaminantes Atmósfericos. (2005). Decreto 30221-S. Obtenido de http://extwprlegs1.fao.org/docs/pdf/cos120292.pdf 
RESOLUÇÃO No 382. (2006). limites máximos de emissão de poluentes atmosféricos para fontes fixas. Recuperado de http://www.mma.gov.br/port/conama/ res/res06/res38206.pdf

Rojas, H. García, G. (2008). Rol del Tribunal Ambiental Administrativo: protección y prevención en materia ambiental. Ciencias Económicas 26,.1. 91-113.
Verdélio, A. (22,03,2017). Brasil carece de legislação para reúso de água, diz coordenador da ANA. EBC Agência Brasil, Recuperado de: http://agenciabrasil.ebc.com. br/geral/noticia/2017-03/brasil-carece-de-legislacaopara-reuso-de-agua-diz-coordenador-da-ana. 